\title{
TVD schemes for unstructured grids
}

\author{
M.S. Darwish, F. Moukalled * \\ Department of Mechanical Engineering, Faculty of Engineering and Architecture, American University of Beirut, P.O. Box 11-0236, \\ Riad El Solh, Beirut 1107 2020, Lebanon
}

Received 4 March 2002; received in revised form 7 August 2002

\begin{abstract}
A number of approaches have evolved over the last decade for the implementation of total variational diminishing (TVD) schemes within an unstructured grid finite volume method framework. Unfortunately none of these approaches has been comprehensive enough to permit the general implementation of TVD-based schemes in unstructured grids, and/or accurate enough to recover the exact TVD formulation in structured grids. In this paper we propose a simple method that allows the implementation of the full spectrum of TVD schemes in unstructured grids, while recovering their exact formulation on structured grids. Four schemes implemented using this approach, TVD-MINMOD, TVDMUSCL (monotonic upstream-centered scheme for conservation laws, MUSCL), TVD-SUPERBEE, TVD-OSHER, are tested and compared to Bruner's TVD formulation [Parallelization of the Euler equations on unstructured grids, AIAA paper 97-1894, 1995], and to the Barth and Jesperson linear reconstruction scheme [The design and application of upwind schemes on unstructured meshes, AIAA paper 89-0366, 1989] by solving four pure advection problems. Results indicate that the Bruner formulation yields, for the same original TVD scheme, overly diffusive results when compared to the current method. The BJ-MUSCL and TVD-MUSCL are shown to be comparable and more accurate than the OSHER scheme. The SUPERBEE performs best though showing tendency for stepping the modeled profile. In all tests the current method is found to retain the behavior of the structured grid TVD formulation.
\end{abstract}

(c) 2002 Elsevier Science Ltd. All rights reserved.

\section{Introduction}

The last two decades have witnessed a sustained effort by the CFD community to develop robust highresolution (HR) schemes [3-9] for the simulation of advection-dominated flows. Many of these schemes have been implemented on structured grids within the framework of finite volume methods. The main ingredients common to all these schemes are a high order profile for the reconstruction of cell face values from cell averages, combined to a monotonicity criterion. The high order reconstruction is usually based on an upwind biased, sometimes symmetric, high order interpolation profile [10-12]. To satisfy monotonicity, a number of

\footnotetext{
${ }^{*}$ Corresponding author. Tel.: +961-3-831-432; fax: +961-1744-462.

E-mail addresses: darwish@aub.edu.lb (M.S. Darwish), memouk@aub.edu.lb (F. Moukalled).
}

concepts have been proposed over the years [13,14], all within a structured grid framework. In the flux corrected transport (FCT) approach [15-18], a first order accurate monotone scheme is converted to a HR scheme by adding limited amounts of anti-diffusive flux. In the monotonic upstream-centered scheme for conservation laws (MUSCL) of Van Leer [19], monotonicity is enforced through a limiter function applied to a piecewise polynomial flux reconstruction procedure. Harten [20] expressed monotonicity as a measure of discrete variation in the solution fields, hence the name total variational diminishing (TVD). This criterion was then expressed as a flux limiter by Sweby using the $r-\psi$ diagram [21]. In the Leonard's approach [4,22,23], the monotonicity criterion is presented using a relation between a normalized face value, $\tilde{\phi}_{f}$, and a normalized upwind value, $\tilde{\phi}_{C}$. While on the conceptual level the above-mentioned monotonicity criteria can be shownto be related and sometimes equivalent, implementation-wise they are very different. However within the framework 


\begin{tabular}{|llll}
\hline \multicolumn{2}{|l}{ Nomenclature } & & \\
$f($ ) & functional relationship & $\nabla$ & gradient operator \\
$r$ & Sweby's $r$-factor & $\bar{\nabla}$ & $\begin{array}{l}\text { linearly Interpolated gradient } \\
\text { position vector }\end{array}$ \\
$u, v$ & velocity components in the $x$ and $y$ directions & $\Delta r$ & \multicolumn{2}{l}{} \\
$\bar{u}, \bar{v}$ & averaged control volume face velocities & \multicolumn{2}{l}{ Subscripts } \\
$\mathbf{v}$ & velocity vector & $f$ & refers to a control volume face \\
$R(~)$ & reconstructed polynomial & $C$ & central grid point \\
Greek & symbols & $D$ & downstream grid point \\
$\Gamma$ & diffusion coefficient & $F$ & neighboring cell point \\
$\phi$ & general dependent variable & $P$ & main grid point \\
$\rho$ & density & $U$ & upstream grid point \\
$\psi$ & Sweby's flux limiter & $\sim$ & refers to normalized variable
\end{tabular}

of structured grids these differences have not translated into increased difficulties in implementation.

For unstructured grids the situation is more complicated and HR schemes are not as advanced as for structured grids [24-26]. This is specifically due to the difficulty in implementing and enforcing a monotonicity criterion that relies on logical or directional nextneighbor information, which is readily available in structured grids but missing in unstructured grids. To circumvent this difficulty a number of approaches have evolved, with varying degrees of success, based on different monotonicity criteria, such as the FCT [14-17], the flux difference splitting concepts [27,28], or the MUSCL approach [29-32]. The MUSCL-based technique developed by Barth and Jespersen [2] (and ammended in $[33,34]$ ), by modifying the Spekreijse [35] definition of monotonicity to bound the cell face values rather than the cell nodal values, is currently the most popular and successful approach for the implementation of HR schemes in unstructured grids [36-44], partly because of its simplicity. Unfortunately, most of the limiters developed for structured grids cannot be implemented using the BJ technique as it is restricted to schemes where the base high order profile uses a cell based gradient, which is basically equivalent to the FROMM scheme [19], whose bounded version is equivalent to the MUSCL scheme. In one dimension the BJ scheme can be shown to be equivalent to the TVDMUSCL scheme [45]. Bruner [1,45] suggested a more general approach to bound convective schemes. In this approach he used the Sweby $r-\psi$ diagram with a modified $r$ factor defined for unstructured grids. Unfortunately his modification did not recover the exact $r$ factor on structured grids. In this paper we present a valid reformulation of the $r$ factor for unstructured grids that yields the exact TVD formulation on structured grids.

In what follows the formulation of TVD schemes is presented for structured grids following the $r-\psi$ diagram of Sweby. The modification of Bruner to the $r$-factor is then described before detailing the new $r$-formulation. Spekreijse's criterion is then presented and the BJ technique described. Finally, the new method is compared to the Bruner implementation and the BJ scheme. For that purpose, four TVD schemes (MINMOD [20], OSHER [46], TVD-MUSCL [19], and SUPERBEE [47]), implemented using the new formulation, are tested by solving a number of pure advection problems.

\section{TVD schemes}

Following Roe [47], the face value $\phi_{i+1 / 2}$ of a TVD scheme is written as the sum of a diffusive first order upwind term and an anti-diffusive one. The anti-diffusive part is multiplied by the flux limiter function, $\psi(r)$, which is a non-linear function of $r$, the upwind ratio of consecutive gradients of the solution, defined as (without loss of generality, we assume the velocity at the face $\left.v_{i+1 / 2}>0\right)$ :

$r_{i+1 / 2}=\frac{\phi_{i}-\phi_{i-1}}{\phi_{i+1}-\phi_{i}}$

leading to the flux-limited scheme:

$\phi_{i+1 / 2}=\phi_{i}+\frac{1}{2} \psi\left(r_{i+1 / 2}\right)\left(\phi_{i+1}-\phi_{i}\right)$

Using a flux limiter, $\psi(r)$, which is simply a linear function of $r$, different high order schemes can be written in the form of Eq. (2). For example for, $\psi$ equal to $r$, the second order upwind (SOU) scheme is obtained. Other schemes can be similarly formulated:

DOWNWIND scheme $\psi(r)=2$

CD scheme $\psi(r)=1$

SOU scheme $\psi(r)=r$

FROMM scheme $\psi(r)=\frac{1+r}{2}$ 
TVD schemes can also be formulated in a form slightly different from the one given by Eq. (2), as in:

$\phi_{i+1 / 2}=\phi_{i}+\frac{1}{2} \psi\left(r_{i+1 / 2}^{\prime}\right)\left(\phi_{i}-\phi_{i-1}\right)$

in this case the $r$ term, now denoted by $r^{\prime}$, is defined as

$r_{i+1 / 2}^{\prime}=\frac{\phi_{i+1}-\phi_{i}}{\phi_{i}-\phi_{i-1}}=\frac{1}{r_{i+1 / 2}}$

the relation between the two formulations is given by

$\frac{1}{r_{i+1 / 2}^{\prime}} \psi^{\prime}\left(r_{i+1 / 2}^{\prime}\right)=\psi\left(r_{i+1 / 2}\right)$

taking for example the SOU scheme where $\psi(r)=r$, the equivalent flux limiter function for Eq. (4) becomes

$\psi_{\mathrm{SOU}}^{\prime}\left(r^{\prime}\right)=r^{\prime} \psi_{\mathrm{SOU}}(r)=\frac{1}{r} r=1$

This can be demonstrated by deriving $\phi_{i+1 / 2}$ for the SOU scheme:

$$
\begin{aligned}
\phi_{i+1 / 2} & =\phi_{i}+\frac{1}{2} \psi_{\mathrm{SOU}}\left(r_{i+1 / 2}\right)\left(\phi_{i+1}-\phi_{i}\right) \\
& =\phi_{i}+\frac{1}{2} r_{i+1 / 2}\left(\phi_{i+1}-\phi_{i}\right) \\
& =\phi_{i}+\frac{1}{2} \frac{\left(\phi_{i}-\phi_{i-1}\right)}{\left(\phi_{i+1}-\phi_{i}\right)}\left(\phi_{i+1}-\phi_{i}\right) \\
& =\frac{3}{2} \phi_{i}-\frac{1}{2} \phi_{i-1} \\
\phi_{i+1 / 2} & =\phi_{i}+\frac{1}{2} \psi_{\mathrm{SOU}}^{\prime}\left(r_{i+1 / 2}\right)\left(\phi_{i}-\phi_{i-1}\right) \\
& =\phi_{i}+\frac{1}{2}\left(\phi_{i}-\phi_{i-1}\right)=\frac{3}{2} \phi_{i}-\frac{1}{2} \phi_{i-1}
\end{aligned}
$$

In this work the formulation of TVD schemes will be performed using Eq. (2). Following Sweby [21], these schemes may be plotted along with the TVD monotonicity region on an $r-\psi$ diagram (see Fig. 1(a)). Using this diagram, it is simple to grasp the formulation of TVD schemes: any flux limiter function, $\psi(r)$, formulated to lie within the TVD monotonicity region yields a

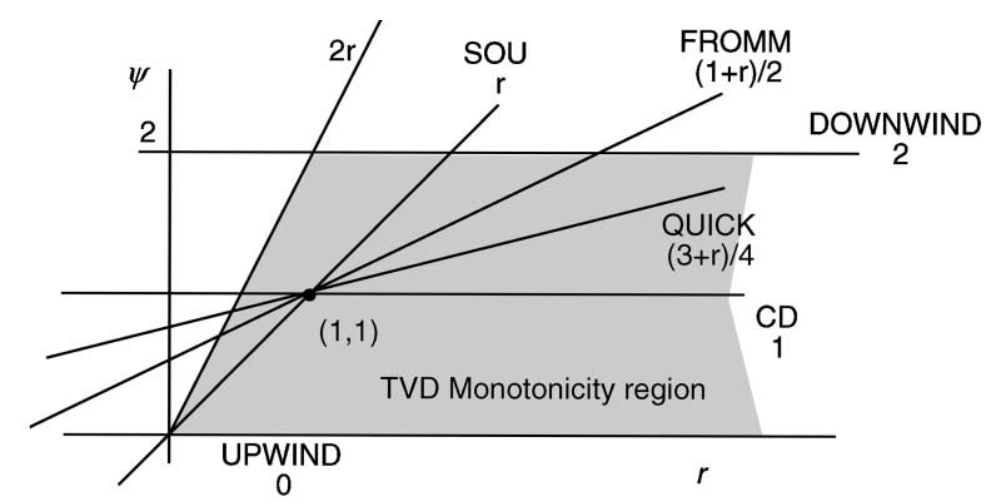

(a)

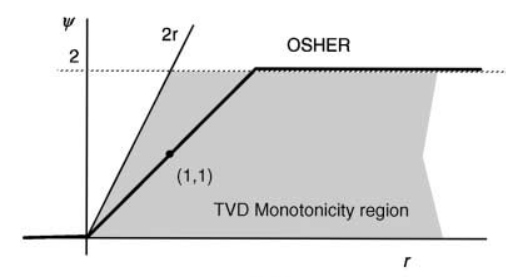

(b)

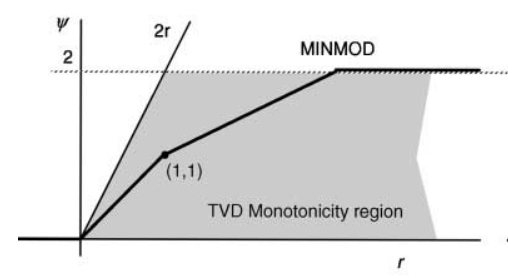

(d)

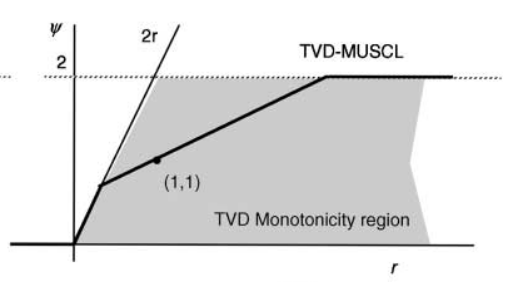

(c)

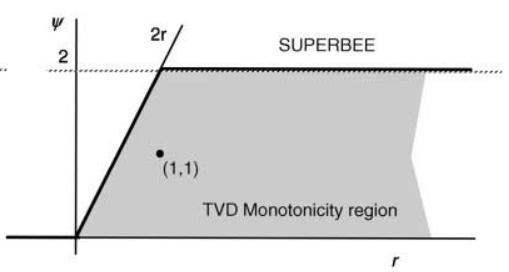

(e)

Fig. 1. (a) High order and TVD monotonicity region on Sweby's diagram, (b)-(e) TVD schemes in Sweby $r-\psi$ diagram. 
TVD scheme. Sweby has also shown that for second order schemes the flux limiter function, $\psi(r)$, has to pass through point $(1,1)$. A number of TVD schemes are shown in Fig. 1(b)-(e), and formulated as

SUPERBEE limiter $\psi(r)=\max (0, \min (1,2 r), \min (2, r)$, MINMOD limiter $\psi(r)=\max (0, \min (1, r))$

OSHER limiter $\psi(r)=\max (0, \min (2, r))$

MUSCL limiter $\psi(r)=\frac{r+|r|}{1+|r|}$

Since the index-based notation used above is not suitable for unstructured grids, the more appropriate notation, shown in Fig. 2(a) and (b), is adopted. As shown in Fig. 2(b), nodes $C$ and $D$ are defined as the Upwind and Downwind nodes around face $f$, and the virtual $U$ node is defined as the node upwind of the $C$ node.

Using this notation Eq. (2) is rewritten as

$\phi_{f}=\phi_{C}+\frac{1}{2} \psi\left(r_{f}\right)\left(\phi_{D}-\phi_{C}\right)$

and the $r$ ratio becomes
$r_{f}=\frac{\phi_{C}-\phi_{U}}{\phi_{D}-\phi_{C}}$

It is clear that the main difficulty in implementing TVD schemes in unstructured grids lies in the need for defining a 'virtual' $U$ node.

\subsection{Bruner $r$ modification}

Bruner $[1,45]$ proposed the following modification to the definition of the $r$-factor for TVD schemes:

$r_{f}=\frac{\phi_{C}-\phi_{U}}{\phi_{D}-\phi_{C}} \approx \frac{2\left(\phi_{f}-\phi_{C}\right)}{\phi_{D}-\phi_{C}}=\frac{2 \Delta \vec{r} \cdot(\nabla \phi)_{C}}{\phi_{D}-\phi_{C}}$

This approximation is clearly inconsistent and when brought back into one dimension does not recover the TVD condition, as Eq. (11) becomes:

$$
\begin{aligned}
r_{f} & =\frac{\phi_{C}-\phi_{U}}{\phi_{D}-\phi_{C}} \neq r_{f, \text { bruner }}=\frac{2 \Delta \vec{r} \cdot(\nabla \phi)_{C}}{\phi_{D}-\phi_{C}} \\
& =\frac{\phi_{D}-\phi_{U}}{\phi_{D}-\phi_{C}}
\end{aligned}
$$

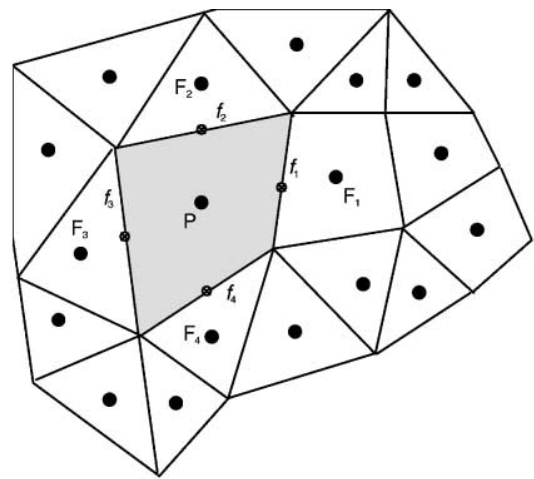

(a)

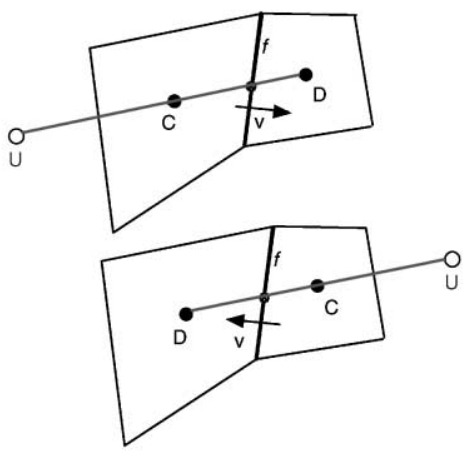

(b)

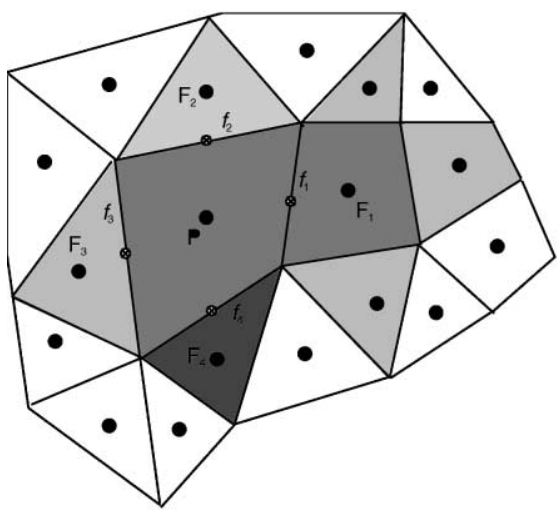

(c)

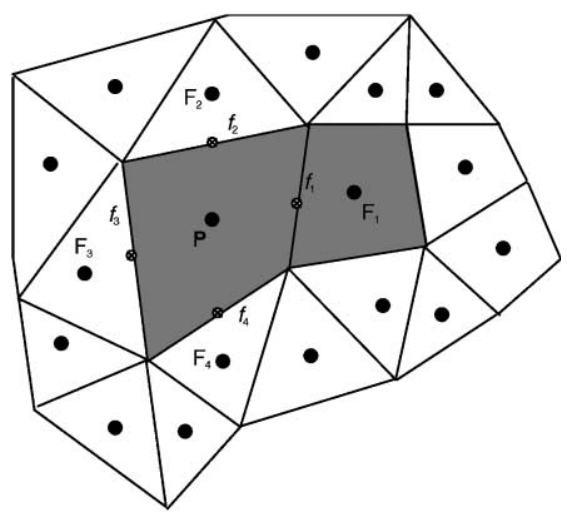

(d)

Fig. 2. (a) Unstructured grid notation, (b) advection node notation, (c) extended stencil for face gradient, and (d) compact stencil for face gradient. 


\subsection{Exact $r$ formulation}

A better formulation for $r$ in unstructured grids can still be derived. Returning to the definition of $r$, we can write:

$$
\begin{aligned}
r_{f} & =\frac{\phi_{C}-\phi_{U}}{\phi_{D}-\phi_{C}}=\frac{\phi_{D}+\left(\phi_{C}-\phi_{U}\right)-\phi_{D}}{\phi_{D}-\phi_{C}} \\
& =\frac{\left(\phi_{D}-\phi_{U}\right)-\left(\phi_{D}-\phi_{C}\right)}{\phi_{D}-\phi_{C}}
\end{aligned}
$$

Noting that values for $\phi_{D}$ and $\phi_{C}$ represent the values of the nodes straddling the interface and thus are readily available for unstructured grid. Therefore, the $r$-values would be computable if the term involving $\phi_{U}$ could be replaced by a known term. In this case

$$
\left(\phi_{D}-\phi_{U}\right)=\nabla \phi_{C} \cdot \mathbf{r}_{U D}=\left(2 \nabla \phi_{C} \cdot \mathbf{r}_{C D}\right)
$$

where $\mathbf{r}_{C D}$ is the vector between the nodes ' $C$ ' and ' $D$ ', and $\mathbf{r}_{U D}$ is the vector between nodes ' $D$ ' and the virtual node ' $U$ ', representing the node Upstream of node ' $C$ ' (see Fig. 2(b)). Node ' $U$ ' is chosen such that it lies along the line joining nodes ' $D$ ' and ' $C$ ' with ' $C$ ' at the center of the ' $U D$ ' segment. Other positions of ' $U$ ' could also be chosen, but with a loss of accuracy as the nodal gradient yields a second order accuracy only when the difference is centered at node ' $C$ '.

The formulation of $r$ becomes

$$
\begin{aligned}
r_{f} & =\frac{\left(2 \nabla \phi_{C} \cdot \mathbf{r}_{C D}\right)-\left(\phi_{D}-\phi_{C}\right)}{\phi_{D}-\phi_{C}} \\
& =\frac{\left(2 \nabla \phi_{C} \cdot \mathbf{r}_{C D}\right)}{\phi_{D}-\phi_{C}}-1
\end{aligned}
$$

which can be easily computed for unstructured grids.

\subsection{Gradient interpolation}

Another important aspect of the TVD implementation resides in the interpolation of gradients to the cell faces. Gradients at the cell faces are used in the discretization of the convection term when using HR schemes and are usually obtained by a weighted interpolation from the neighboring cell gradients. A simple weighted interpolation leads to an extended stencil as shown in Fig. 2(c), the stencils of the cell gradients computed using the Gauss theorem or least squares involving the neighboring cell nodes are added to yield the face gradient extended stencil. A better method is to force the face gradient along the PN direction (Fig. 2(d)) to be directly computed from the cell nodes in a manner similar to the Rhie-Chow interpolation [48] for pressure gradients. In this case the cell face gradient along $\mathrm{PN}$ is more compact and accurate, and is given by

$$
(\nabla \phi)_{f}=\overline{(\nabla \phi)}_{f}+\frac{\left(\phi_{F}-\phi_{P}\right)}{\left\|\mathbf{r}_{P F}\right\|} \mathbf{e}_{P F}-\left(\overline{(\nabla \phi)}_{f} \cdot \mathbf{e}_{P F}\right) \mathbf{e}_{P F}
$$

where $\overline{(\nabla \phi)}_{f}$ is the gradient interpolated from the two adjacent cell gradients, $\mathbf{r}_{P F}$ the vector between the nodes $P$ and $F$ (where $F=F 1$, or $F 2, \ldots$ ), and $\mathbf{e}_{P F}$ is a unit vector in the direction of $\mathbf{r}_{P F}$. What is achieved with this formulation is a reduction in the stencil of the face gradient along the $P F$ direction. The stencil for $\overline{(\nabla \phi)}_{f}$, shown in Fig. 2(c), is basically the union of the stencils of the $P$ and $F$ cells, whereas that for $(\nabla \phi)_{f}$ along the $P F$ direction is formed of the $P$ and $F$ cells only, (Fig. 2(d)).

This modification was found to be especially important for HR schemes that are defined as a function of face gradients such as the SOU scheme.

\section{Barth and Jesperson scheme}

BJ [2] followed a different approach in enforcing the monotonicity criterion. In their approach the Speikreijse monotonicity criterion [35] was modified to apply it for the reconstruction of $\phi$ within the control volume, i.e. the condition was changed to state that the values of the reconstructed polynomial within the control volume should not exceed the maximum and minimum values at the neighbors of the control volume.

Using the notation of Fig. 2(a) the Speikreijse criterion written as

$\min \left(\phi_{N}\right) \leqslant \phi_{P} \leqslant \max \left(\phi_{N}\right) \forall N \in \operatorname{Neighbors}(P)$

was modified to:

$\min \left(\phi_{N}, \phi_{P}\right) \leqslant R_{P}\left(\mathbf{r}_{j}\right) \leqslant \max \left(\phi_{N}, \phi_{P}\right) \forall N \in \operatorname{Neighbors}(P)$

where $j$ is some point within the control volume $P$, and $R$ is the reconstruction operator given by

$R_{P}\left(\mathbf{r}_{j}\right)=\phi_{P}+\psi_{j} \nabla \phi_{P} \cdot\left(\mathbf{r}_{j}-\mathbf{r}_{P}\right)$

where $\psi$ is the flux limiter, and $\mathbf{r}$ the position vector. Instead of enforcing the condition of Eq. (2) over the control volume, it is enforced at the cell faces integration points, thus changing Eq. (19) into:

$\min \left(\phi_{N}, \phi_{P}\right) \leqslant R_{P}\left(\mathbf{r}_{f}\right) \leqslant \max \left(\phi_{N}, \phi_{P}\right) \forall N \in \operatorname{Neighbors}(P)$

where $f$ is a cell face centroid. The computation of the value of the limiter $\psi$ is performed in two steps as follows.

Step 1: The reconstruction polynomial is evaluated at each cell face integration point in order to determine the value of $\psi_{f}$ that satisfies the relation: 


$$
\begin{aligned}
\min \left(\phi_{N}, \phi_{P}\right) \leqslant R_{P}\left(\mathbf{r}_{f}\right) & =\phi_{P}+\psi \nabla \phi_{P} \cdot\left(\mathbf{r}_{f}-\mathbf{r}_{P}\right) \\
& \leqslant \max \left(\phi_{N}, \phi_{P}\right)
\end{aligned}
$$

which can be rewritten as

$$
\psi_{f}= \begin{cases}\Phi\left(\frac{\max \left(\phi_{N}, \phi_{P}\right)-\phi_{P}}{\nabla \phi_{P} \cdot\left(\mathbf{r}_{f}-\mathbf{r}_{P}\right)}\right) & \phi_{f}>\phi_{P} \\ \Phi\left(\frac{\phi_{P}-\min \left(\phi_{N}, \phi_{P}\right)}{\nabla \phi_{P} \cdot\left(\mathbf{r}_{f}-\mathbf{r}_{P}\right)}\right) & \phi_{f}<\phi_{P} \\ 1 & \phi_{f}=\phi_{P}\end{cases}
$$

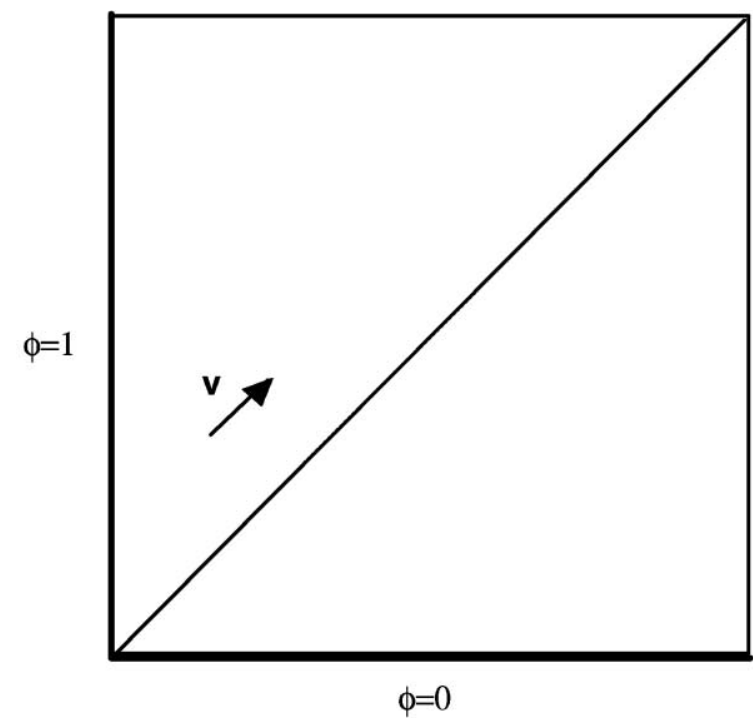

(a)

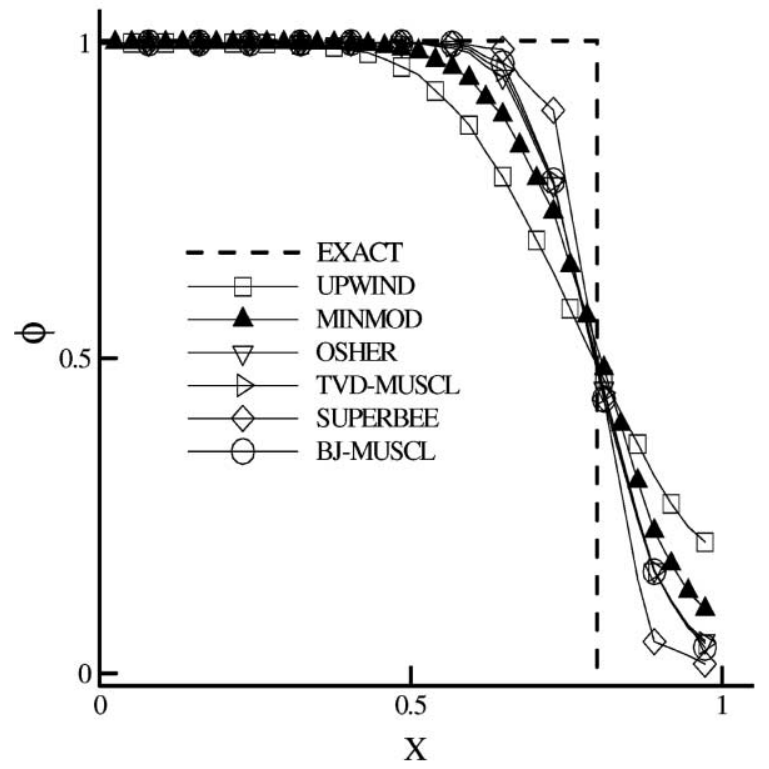

(c)

Fig. 3. Convection of a step profile: (a) physical domain, (b) dense grid used, (c) $\phi$ profile at $y=0.8$ using coarse grid, (d) $\phi$ profile at $y=0.8$ using dense grid. where

$\Phi(x)=\min (x, 1)$

Step 2: The value of the limiter is computed as the minimal value of $\psi$ over all cell faces, i.e.:

$\psi_{P}=\min \left(\psi_{f}\right)$

Because of convergence problems found with the limiter, Venkatakrishnan $[33,40]$ proposed a modified version of

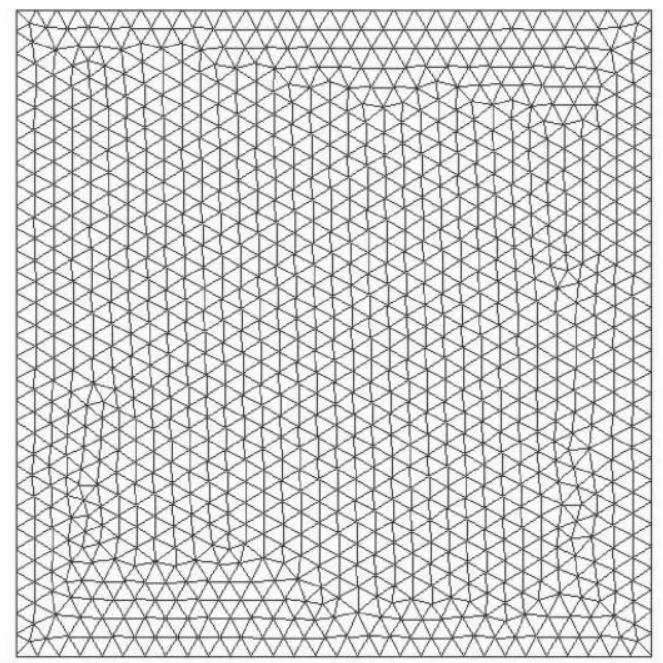

(b)

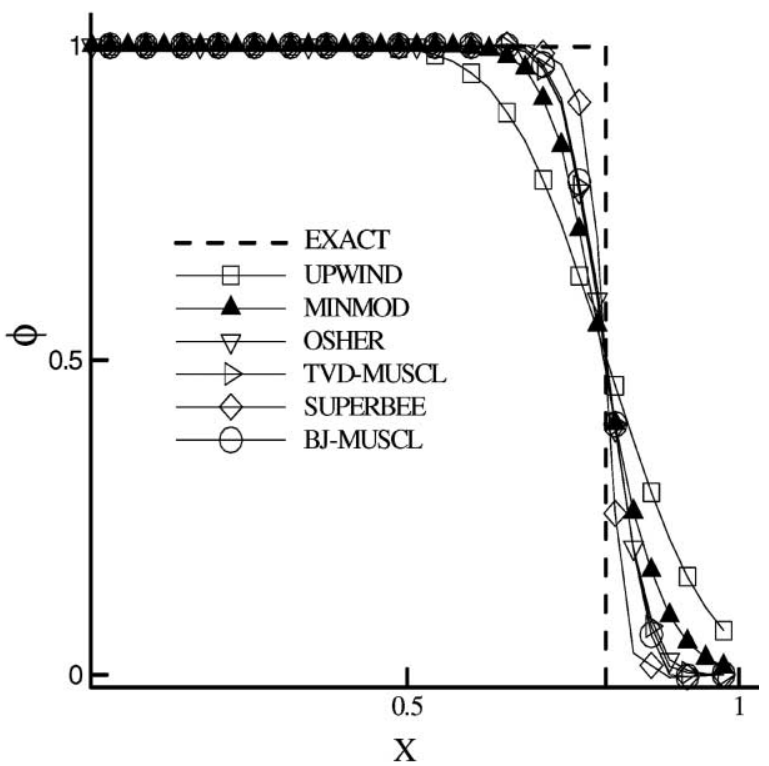

(d) 
the original limiter. The modified limiter is based on the differentiable function:

$\Phi(x)=\frac{x^{2}+2 x+\varepsilon^{2}}{x^{2}+x+2+\varepsilon^{2}}$

The role of the constant, $\varepsilon^{2}$, being to deactivate the limiter in smooth flow regions, with its value specified as $\varepsilon^{2}=(K h)^{3}$ where $K$ is a user-specified constant, and $h$ a local mesh size.

It is worth noting that for a one dimensional discretization, the Barth and Jesperson scheme can be shown to be equivalent to the TVD-MUSCL scheme, and thus in this special case could be represented graphically using the Sweby diagram of Fig. 1(c).

\section{Test problems}

The validity of the newly proposed $r$-factor formulation in unstructured grids is demonstrated in this section. For that purpose four TVD schemes are implemented using the Bruner and the new formulations. The schemes are then used in solving four pure convection test problems: advection of a step profile, advection of a sinusoidal profile, advection of a doublestep profile, and the Smith and Hutton problem [49]. Results obtained using the schemes are compared against those obtained using the $\mathrm{BJ}$ linear reconstruction scheme and the first order upwind scheme.

\subsection{Advection of a step profile}

Fig. 3(a) shows the well known benchmark test problem consisting of a pure advection of a transverse step profile imposed at the inflow boundaries of a square computational domain. Two unstructured mesh systems consisting of 862 and 2094 cells (illustrated in Fig. 3(b)) were used. The governing conservation equation for the problem is simply:

$$
\nabla \cdot(\rho \mathbf{v} \phi)=0
$$

where $\phi$ is the dependent variable and $\mathbf{v}=1 \mathbf{i}+1 \mathbf{j}$ is the Cartesian velocity vector. The computed values of $\phi$ using the upwind scheme, the OSHER, the MINMOD, the MUSCL and the SUPERBEE schemes, implemented using the exact $r$-formulation, in addition to the BJ scheme are shown in Fig. 3(c) and (d) for the coarse and fine grids respectively. As is the case for structured grids [5] the MINMOD is the most diffusive, the BJ-MUSCL and TVD-MUSCL schemes yield comparable results that are better than the OSHER scheme. The performance of the upwind scheme is worse in unstructured grids than for structured grids because no flow can be aligned with the grid lines. As expected the SUPERBEE yields the best results because it is a highly compressive scheme. All results are devoid of over/undershoots.
Performance-wise the TVD and BJ implementations required about the same computational cost per iteration, TVD schemes generally did not experience any flattening of the convergence rate below a residual of $10^{-6}$ except for the SUPERBEE scheme, while the BJ scheme experienced oscillations of the residual around $10^{-6}$. This was inconsequential with respect to the results.

In Fig. 4, the TVD-MUSCL scheme profiles generated using the current formulation are compared against profiles obtained following Bruner's formulation on both coarse (Fig. 4(a)) and dense (Fig. 4(b)) grid systems. As depicted, Bruner's scheme results are over diffusive due to the approximation introduced in the formulation of $r$. The same trend was observed with all the other schemes and deemed unnecessary to be reported for compactness.
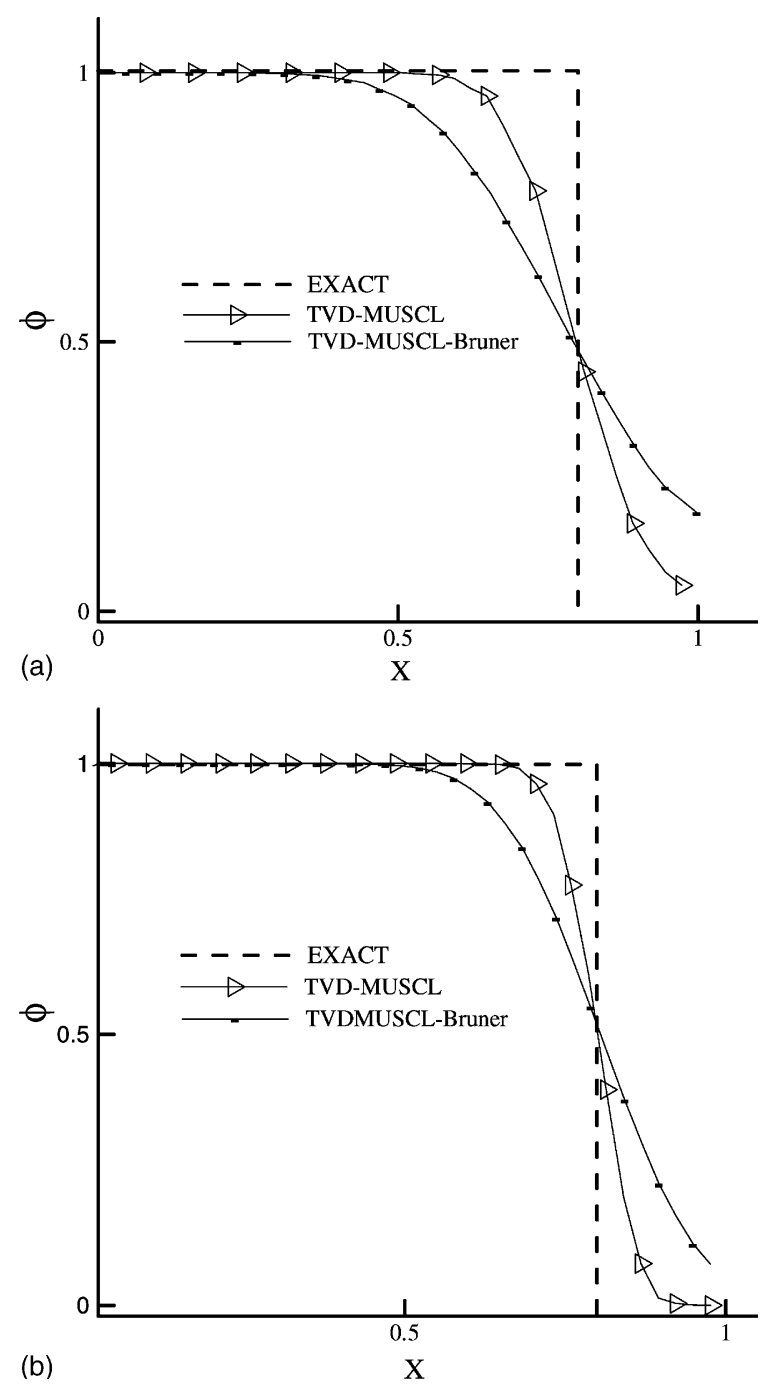

Fig. 4. Comparison of $\phi$ profiles at $y=0.8$ using MUSCL scheme implemented via the new TVD formulation against Bruner's formulation [1] over (a) coarse and (b) dense grid systems for the pure convection of a step profile problem. 


\subsection{Advection of a sinusoidal profile}

This problem is similar to the previous one in geometry except that a sinusoidal profile is used. The sinusoidal profile involves steep and smooth regions, as well as an extremum point, making its simulation much more demanding than the simple step profile. The profile is given as

$\phi= \begin{cases}\sin \left(\frac{\pi}{2} \max \left(1-\frac{\operatorname{abs}(y-0.1707)}{0.1707}, 0\right)\right) & 0 \leqslant y \leqslant 0.3414 \\ 0 & \text { otherwise }\end{cases}$

The problem is depicted in Fig. 5(a), the same meshes as the step-profile problem were used. Results are shown in Fig. 5(b) and (c) for the coarse and fine meshes respec- tively. As expected all the schemes suffer from a substantial decrease in the numerical extremum, with its value decreasing down to 0.48 for the UPWIND scheme. The SUPERBEE preserves on the coarse mesh more of the extremum value $(0.83)$, and experience no loss in extremum on the fine mesh. The BJ-MUSCL, TVDMUSCL and OSHER schemes results are much better than those of the UPWIND scheme and the MINMOD scheme, while still experiencing on the coarse and fine meshes a decrease in the extrema down to 0.68 and 0.92 respectively.

\subsection{Advection of a double-step profile}

A double-step profile is imposed at inlet to the square domain depicted in Fig. 6(a). The profile is given as
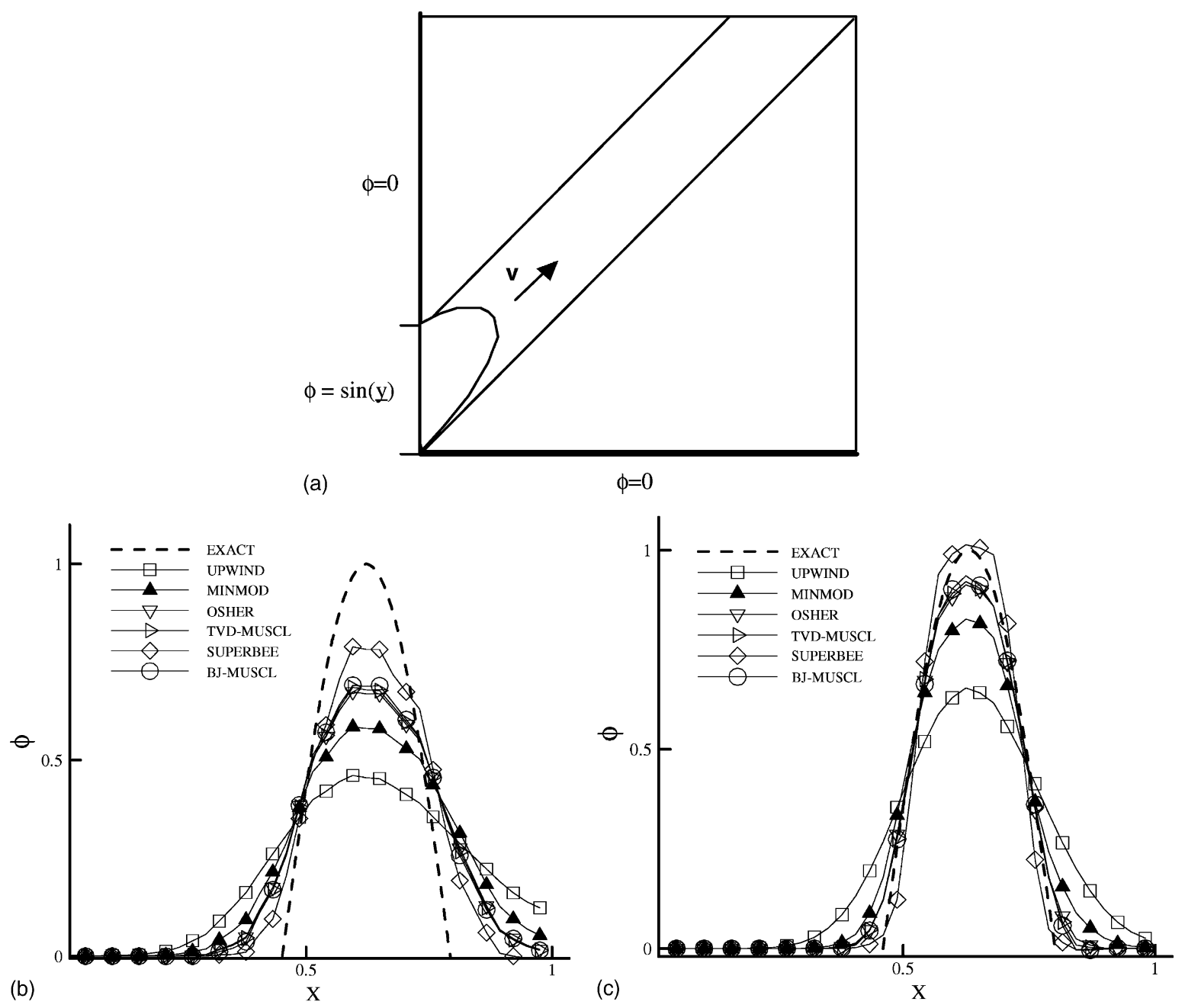

Fig. 5. Convection of a sinusoidal profile: (a) physical domain, (b) $\phi$ profile at $y=0.8$ using coarse grid, (c) $\phi$ profile at $y=0.8$ using dense grid. 
$\phi= \begin{cases}1 & 0 \leqslant y \leqslant 0.3 \\ 0 & y>0.3\end{cases}$

The same meshes as for the step-profile problem were used. Results are shown for the coarse and fine meshes in Fig. 6(b) and (c) respectively. As expected all schemes suffer from an important decrease in the numerical extremum on the coarse mesh, however on the fine mesh the SUPERBEE, OSHER, TVD-MUSCL and BJMUSCL preserve the extremum of 1.0. The profiles obtained by the MINMOD and UPWIND schemes are however of lower quality.

\subsection{Smith and Hutton problem}

In the fourth test problem, shown schematically in Fig. 7(a) along with an illustrative grid used (Fig. 7(b)), a step discontinuity at $x=-0.5$ is convected clockwise from the inlet plane $(x<0, y=0)$ to the outlet plane $(x>0, y=0)$ by a rotational velocity field given by

$\mathbf{v}=\left\{\begin{array}{l}u \\ v\end{array}\right\}=\left\{\begin{array}{c}2 y\left(1-x^{2}\right) \\ -2 x\left(1-y^{2}\right)\end{array}\right\}$

The use of the above equation, denoted by the "point formula', to compute the convective fluxes yielded a non-conservative velocity field, i.e. continuity was not satisfied over each cells. This is clearly demonstrated in the continuity residuals' map depicted in Fig. 7(c). The reason for this behavior is that the cell face velocities in the discretized equation are assumed, when using the point formula, to be constant through out the face, which is clearly wrong. In order to satisfy continuity
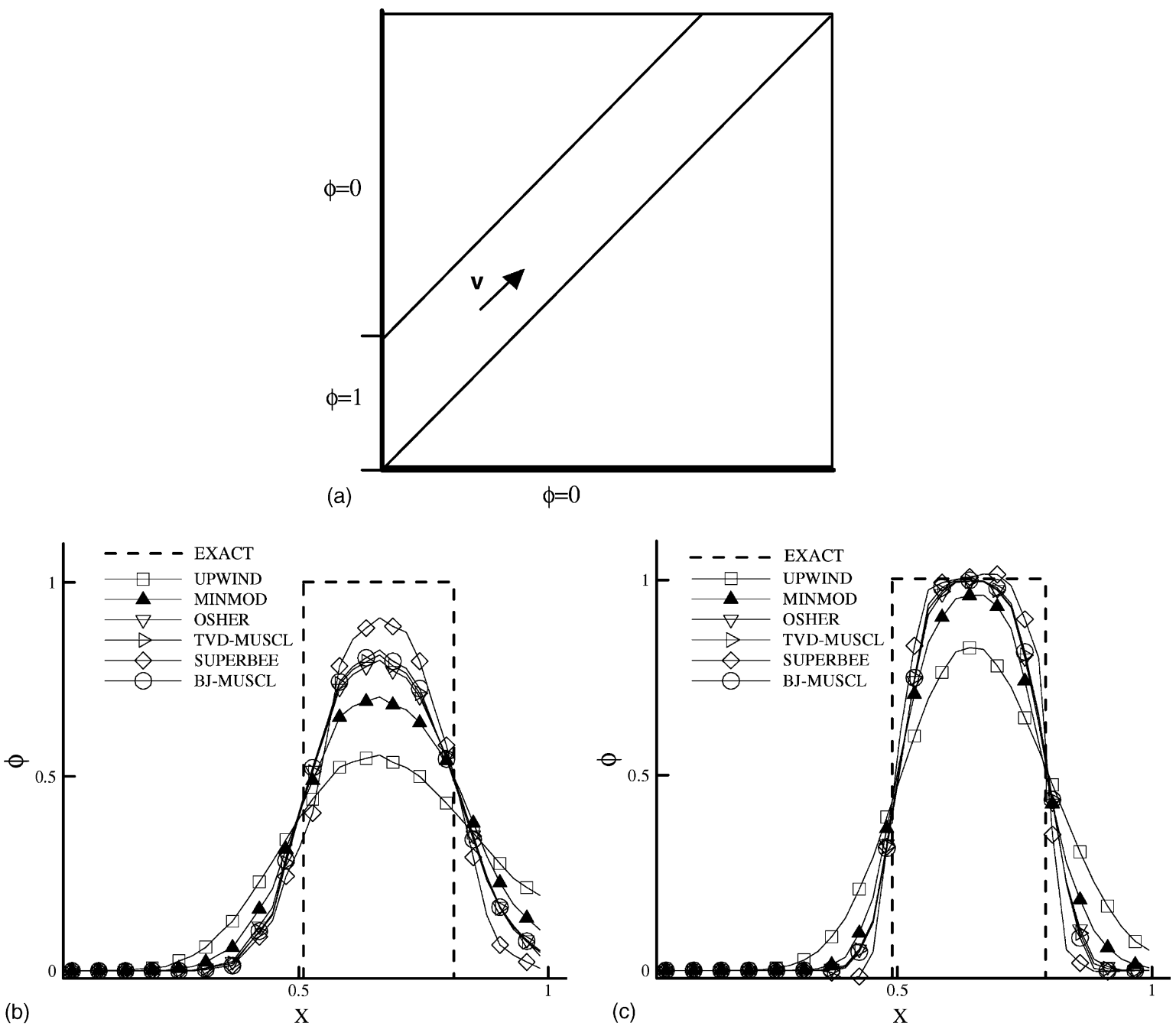

Fig. 6. Convection of a double-step profile: (a) physical domain, (b) $\phi$ profile at $y=0.8$ using coarse grid, (c) $\phi$ profile at $y=0.8$ using dense grid. 

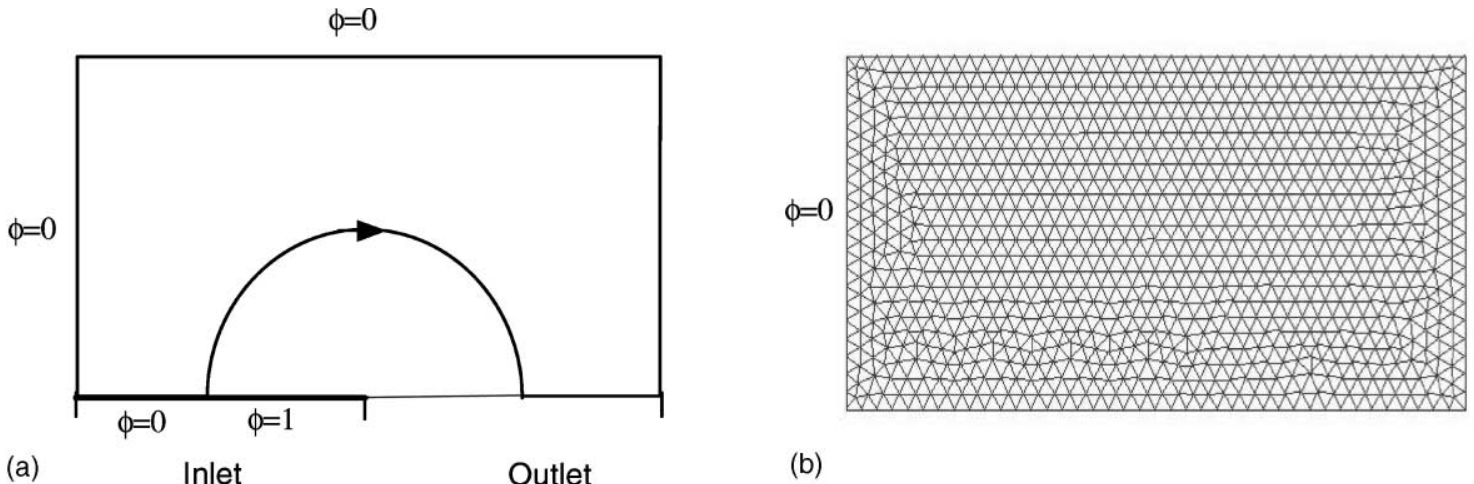

(a)

Outlet

(b)

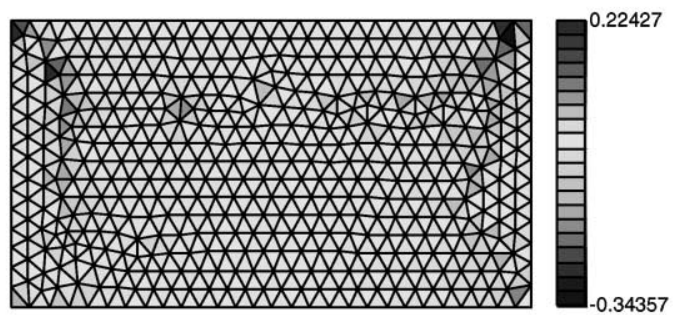

(c)

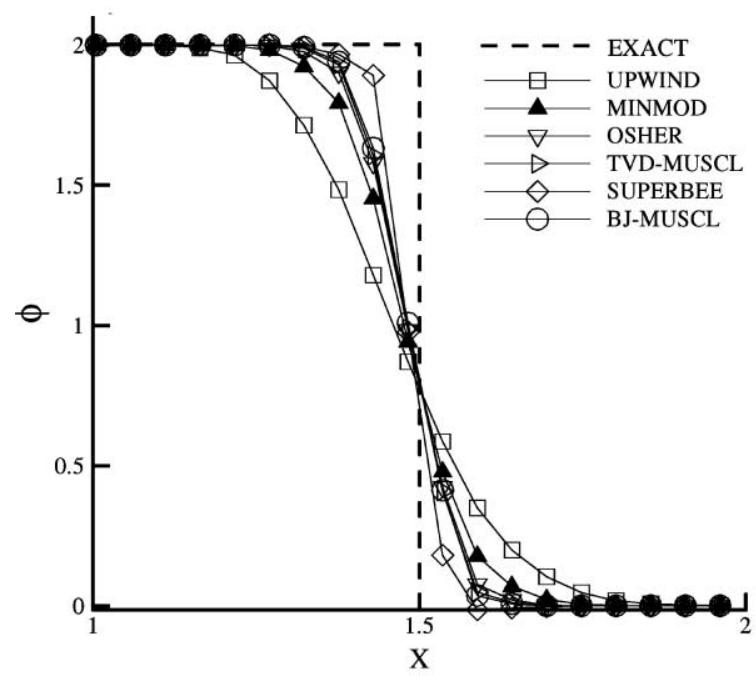

(e)

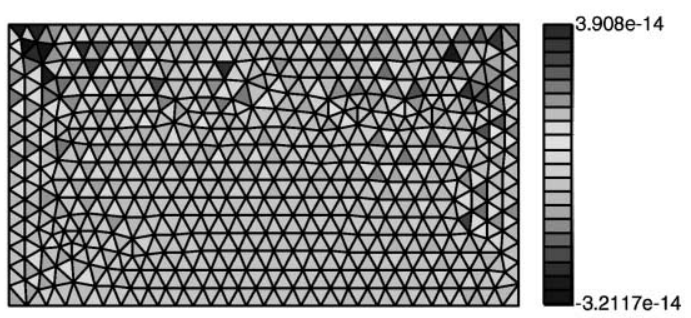

(d)

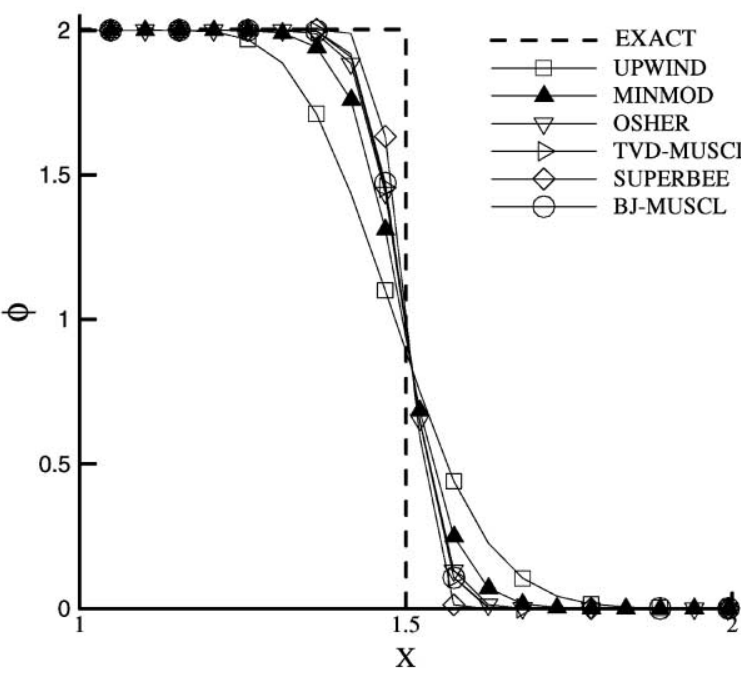

(f)

Fig. 7. Smith Hutton problem: (a) physical domain, (b) dense grid used, (c) divergence error over the domain using the "point formulation", (d) divergence error over the domain using the "integral formulation", (e) $\phi$ profile at exit from the domain ( $y=0$ ) using coarse grid, (f) $\phi$ profile at exit from the domain $(y=0)$ using dense grid.

over each cell the above equations should be integrated over the cell faces to yield the respective face fluxes. The resulting equation is denoted here by the 'integration formula'. Integrating Eq. (30) over a cell face yielded the following equations for the $x$ and $y$ face fluxes:

$$
\int_{y_{n_{1}}}^{y_{n_{2}}} \int_{x_{n_{1}}}^{x_{n_{2}}} u \mathrm{~d} x \mathrm{~d} y=\int_{y_{n_{1}}}^{y_{n_{2}}} \int_{x_{n_{1}}}^{x_{n_{2}}} 2 y\left(1-x^{2}\right) \mathrm{d} x \mathrm{~d} y
$$

over a general cell face defined by

$y=m x+n$ with $m=\frac{y_{n_{2}}-y_{n_{1}}}{x_{n_{2}}-x_{n_{1}}}, \quad n=y_{n_{2}}-m x_{n_{2}}$ 
Integrating equation (31) from node 1 to node 2 , one gets:

$$
\begin{aligned}
\bar{u}= & \frac{1}{l} \int_{n_{1}}^{n_{2}} u \mathrm{~d} l=\int_{x_{n_{1}}}^{x_{n_{2}}} 2(m x+n)\left(1-x^{2}\right) \mathrm{d} x \\
= & \frac{1}{l} \int_{x_{n_{1}}}^{x_{n_{2}}}\left(2 m x+2 n-2 m x^{3}-2 n x^{2}\right) \mathrm{d} x \\
= & \frac{1}{l}\left[m x^{2}+2 n x-\frac{1}{2} m x^{4}-\frac{2}{3} n x^{3}\right]_{x_{n_{1}}}^{x_{n_{2}}} \\
= & \frac{1}{l}\left[m\left(x_{n_{2}}^{2}-x_{n_{1}}^{2}\right)+2 n\left(x_{n_{2}}-x_{n_{1}}\right)-\frac{1}{2} m\left(x_{n_{2}}^{4}-x_{n_{1}}^{4}\right)\right. \\
& \left.\quad-\frac{2}{3} n\left(x_{n_{2}}^{3}-x_{n_{1}}^{3}\right)\right]
\end{aligned}
$$

and for the $y$ component we get

$$
\begin{aligned}
\bar{v}= & \frac{1}{l} \int_{n_{1}}^{n_{2}} v \mathrm{~d} l=\int_{x_{n_{1}}}^{x_{n_{2}}}-2 x\left(1-(m x+n)^{2}\right) \mathrm{d} x \\
= & \frac{1}{l} \int_{x_{n_{1}}}^{x_{n_{2}}}\left(-2 x+2 n^{2} x+2 m^{2} x^{3}+4 m n x^{2}\right) \mathrm{d} x \\
= & \frac{1}{l}\left[-x^{2}+n^{2} x^{2}+\frac{1}{2} m^{2} x^{4}+\frac{4}{3} m n x^{3}\right]_{x_{n_{1}}}^{x_{n_{2}}} \\
= & \frac{1}{l}\left[-\left(x_{n_{2}}^{2}-x_{n_{1}}^{2}\right)+n^{2}\left(x_{n_{2}}^{2}-x_{n_{1}}^{2}\right)+\frac{1}{2} m^{2}\left(x_{n_{2}}^{4}-x_{n_{1}}^{4}\right)\right. \\
& \left.\quad-\frac{4}{3} m n\left(x_{n_{2}}^{3}-x_{n_{1}}^{3}\right)\right]
\end{aligned}
$$

where $m$ and $n$ define the equation passing through nodes $n_{1}$ and $n_{2}$, i.e. the cell face. Using the integration formula' to compute the velocity component yielded a continuity satisfying velocity fields as shown by the map displayed in Fig. 7(d).

The boundary conditions for the Smith and Hutton problem [49] are:

$$
\phi= \begin{cases}2 & \text { for }-0.5<x<0 ; y=0 \\ 0 & \text { for }-1<x<-0.5 ; y=0 \\ 0 & \text { for }-1<x<1 ; y=1 \\ 0 & \text { for } x=-1 ; 0<y<1 \\ 0 & \text { for } x=1 ; 0<y<1\end{cases}
$$

No physical diffusion was considered and the problem was solved using two grid systems of size 889 and 1060 cells (Fig. 7(b)). Results are displayed in Fig. 7(e) and (f). As before, for the coarse mesh numerical results obtained with the SUPERBEE are better than those achieved with the other schemes. For the fine mesh the SUPERBEE, OSHER, BJ-MUSCL and TVD-MUSCL scheme profiles are nearly similar. Results from the MINMOD scheme are quite diffusive, but nonetheless better than the UPWIND scheme.

\section{Conclusion}

In this paper, a number of TVD schemes, namely the SUPERBEE, TVD-MUSCL, OSHER, and MINMOD schemes, in addition to the BJ-MUSCL scheme were implemented on unstructured grids. As expected, results for the BJ-MUSCL and TVD-MUSCL were found to be similar. The approach followed was proved to be general and consistent with the Sweby TVD formulation for structured grids. Results for all test problems presented showed that TVD monotonicity was properly enforced for all schemes resulting in oscillation free profiles. The benefit of such an approach are twofold: (i) a wide range of TVD schemes including compressive schemes such as the SUPERBEE scheme can be readily implemented; (ii) improvement to the standard TVD formulation such as extremum preserving algorithms [50] can now be used for unstructured grids.

\section{Acknowledgement}

The financial support provided by the University Research Board of the American University of Beirut is gratefully acknowledged.

\section{References}

[1] C. Bruner, R. Walters, Parallelization of the Euler equations on unstructured grids, AIAA paper 97-1894, 1995.

[2] T. Barth, D.C. Jespersen, The design and application of upwind schemes on unstructured meshes, AIAA paper 890366, 1989.

[3] B.P. Leonard, Simple high accuracy resolution program for convective modeling of discontinuities, International Journal for Numerical Methods in Engineering 8 (1988) 12911319.

[4] B.P. Leonard, The ULTIMATE conservative difference scheme applied to unsteady one-dimensional advection, Computer Methods in Applied Mechanics and Engineering 88 (1991) 17-74.

[5] F. Moukalled, M.S. Darwish, A new bounded-skew central difference scheme-Part I: Formulation and testing, Numerical Heat Transfer, Part B: Fundamentals 31 (1) (1996) 91-110.

[6] H. Gerges, J. McCorquodale, Modeling of flow in rectangular sedimentation tanks by an explicit third-order upwinding techniques, International Journal for Numerical Methods in Fluids 24 (1997) 537-561.

[7] H. Deconinck, P.L. Roe, R. Struijs, A multidimensional generalization of Roe's flux difference splitter for the Euler equations, Computers and Fluids 22 (23) (1993) 215-222.

[8] D. Sidilkover, A genuinely multidimensional upwind scheme and efficient multigrid solver for the compressible Euler equations, ICASE Report No. 94-84, 1994.

[9] A. Jameson, Artificial diffusion, upwind biasing limiters and their effect on accuracy of multigrid convergence in transonic and hypersonic flows, AIAA paper 3359, 1993. 
[10] B.P. Leonard, A stable and accurate convective modeling procedure based on quadratic interpolation, Computer Methods in Applied Mechanics and Engineering 19 (1979) 59-98.

[11] B.P. Leonard, H. Niknafs, Sharp monotonic resolution of discontinuities without clipping of narrow extrema, Computers and Fluids 19 (1991) 141-154.

[12] P. Holnicki, A piecewise-quintic interpolation scheme, Journal of Computational Physics 127 (1996) 316-329.

[13] S.K. Godunov, A difference scheme for numerical computation of discontinuous solution of hydrodynamic equations, Translated from Russian by US joint publication research service, 1969-JPRS 7226, 1959.

[14] R.J. Leveque, Numerical Methods for Conservation Laws, Birhäuser, Germany, 1992.

[15] S.T. Zalesak, Fully multidimensional flux corrected transport algorithms for fluids, Journal of Computational Physics 31 (1979) 335-362.

[16] J.P. Boris, D.L. Book, Flux-corrected transport I. SHASTA, a transport algorithm that works, Journal of Computational Physics 11 (1973) 38-69.

[17] J.P. Boris, D.L. Book, Flux corrected transport III, minimal error FCT algorithms, Journal of Computational Physics 20 (1976) 397-431.

[18] D.L. Book, S.T. Zalesak, Flux corrected transport, in: D.L. Book (Ed.), Finite-Difference Techniques for Vectorized Fluid Dynamics Calculations, Springer Verlag, NY, 1981.

[19] B. Van Leer, Towards the ultimate conservative difference scheme. V. A second-order sequel to Godunov's method, Journal of Computational Physics 32 (1979) 101-136.

[20] A. Harten, High resolution schemes for hyperbolic conservation laws, Journal of Computational Physics 49 (1983) 357-393.

[21] P.K. Sweby, High resolution schemes using flux-limiters for hyperbolic conservation laws, SIAM Journal of Numerical Analysis 21 (1984) 995-1011.

[22] P.H. Gaskell, A.K.C. Lau, Curvature compensated convective transport: SMART, a new boundedness preserving transport algorithm, International Journal for Numerical Methods in Fluids 8 (1988) 617-641.

[23] M.S. Darwish, A new high-resolution scheme based on the normalized variable formulation, Numerical Heat Transfer, Part B: Fundamentals 24 (1993) 353-371.

[24] V. Venkatakrishnan, Perspective on unstructured grid solvers, AIAA Journal 34 (3) (1996) 533-547.

[25] M. Aftomis, N. Kroll, A quadrilateral-based second order TVD method for unstructured adaptive meshes, AIAA Paper 91-0124, 1991.

[26] A.A. Fursenko, D.M. Shaov, E.V. Timofeev, P.A. Voinovich, High resolution schemes in unstructured grids in transient shocked flow simulation, Proceedings of the 13th International Conference On Numerical Methods in Fluid Dynamics, 1993, pp. 250-255.

[27] R. Löhner, K. Morgan, J. Peraire, M. Vahdati, Finite element flux-corrected transport (FEM-FCT) for the Euler and Navier-Stokes equations, International Journal for Numerical Methods in Fluids 7 (1987) 1093-1109.

[28] L. Ferzoui, B. Stoufflet, A class of implicit upwind schemes for Euler simulations with unstructured grids, Journal of Computational Physics (84) (1989) 174-206.
[29] J.A. Desideri, A. Dervieux, Compressible flow solvers using unstructured grids, VKI Lecture Series, 1988-05, van Karman Institute of Fluid Dynamics, Belgium, 1988, pp. $1-115$.

[30] T.J. Batina, Implicit flux-split Euler scheme for unsteady aerodynamic analysis involving unstructured dynamic meshes, AIAA Paper 90-0013, 1990.

[31] V. Venkatakrishnan, T.J. Barth, Application of direct solvers to unstructured meshes, Journal of Computational Physics 105 (1993) 83-91.

[32] N.T. Frink, Upwind schemes for solving the Euler equations on unstructured tetrahedral meshes, AIAA Journal 3 (1) (1992) 70-77.

[33] V. Venkatakrishnan, On the accuracy of limiters and convergence to steady state solutions, AIAA Paper 930880, 1993.

[34] V. Venkatakrishnan, Parallel implicit unstructured grid Euler solvers, AIAA Journal 32 (1994) 19851991.

[35] S. Spekreijse, Multigrid solution of monotone, second order discretization of hyperbolic conservation laws, Mathematics of Computations 45 (1987) 15-21.

[36] T.J. Barth, Recent development in High Order k-Exact Reconstruction on Unstructured Meshes, AIAA paper 930668, January 1993.

[37] A.G. Godfrey, C.R. Mitchell, R.W. Walters, Aspects of spatially high accuracy methods, AIAA Paper 92-0054, 1992.

[38] V. Venkatakrishnan, D.J. Mavriplis, Implicit solvers for unstructured meshes, Journal of computational Physics 105 (1993) 83-91.

[39] W.K. Anderson, A grid generation and flow solution method for Euler equations on unstructured grids, Journal of computational Physics 110 (1994) 23-38.

[40] V. Venkatakrishnana, Convergence to steady state solutions of the Euler equations on unstructured grids with limiters, Journal of Computational Physics 118 (1995) 120130 .

[41] M. Aftosmis, D. Gautibde, T.S. Tavares, Behavior of linear reconstruction techniques on unstructured meshes, AIAA Journal 33 (11) (1995) 2038-2059.

[42] M. Kobayashi, J.M.C. Pereira, J.C.F. Pereira, A secondorder upwind least square scheme for incompressible flows on unstructured hybrid grids, Numerical Heat Transfer, part B: Fundamentals 34 (1) (1998) 39-60.

[43] R.C. Swanson, R. Radespiel, E. Turkel, On some numerical dissipation schemes, Journal of Computational Physics 147 (1998) 518-544.

[44] D.W. Zing, S. De Rango, M. Nemec, T.H. Pulliam, Comparison of several spatial discretizations for the Navier-Stokes equations, AIAA paper 99-3260, 1999.

[45] S. Bruner, Parallelization of the Euler equatons on unstructured grids, Ph.D. thesis, Department of Aerospace engineering, Virginia Polytechnic Institute and State University, 1996.

[46] S.R. Chakravarthy, S. Osher, High resolution applications of the OSHER upwind scheme for the Euler equations, AIAA Paper 83-1943, 1983.

[47] P.L. Roe, Some contributions to the modeling of discontinuous flows, Proceedings of the AMS/SIAM Seminar, San Diego, 1983. 
[48] C.M. Rhie, W.L. Chow, Numerical study of the turbulent flow past and airfoil with trailing edge separation, AIAA Journal 21 (1983) 1525-1532.

[49] R.M. Smith, A.G. Hutton, The numerical treatment of advection: a performance comparison of current methods, Numerical Heat Transfer 5 (1982) 439-461.
[50] M.S. Darwish, F. Moukalled, B-EXPRESS: new Bounded EXtrema PREServing strategy for convective schemes, Numerical Heat Transfer Part B: Fundamentals 37 (2) (2000) 227-246. 